\title{
Identifying Simultaneous Matrix \\ Metalloproteinases/Soluble Epoxide Hydrolase Inhibitors
}

\author{
Ahmed A. El-Sherbeni \\ University of Alberta \\ Rabia Bhatti \\ University of Alberta \\ Fadumo A. Isse \\ University of Alberta \\ Ayman 0.S. El-Kadi ( $\square$ aelkadi@ualberta.ca ) \\ University of Alberta https://orcid.org/0000-0002-8692-0400
}

\section{Research Article}

Keywords: Matrix metalloproteinase, soluble epoxide hydrolase, cardiovascular diseases, extracellular matrix, epoxyeicosatrienoic acids.

Posted Date: October 28th, 2021

DOl: https://doi.org/10.21203/rs.3.rs-991660/v1

License: (9) This work is licensed under a Creative Commons Attribution 4.0 International License. Read Full License

Version of Record: A version of this preprint was published at Molecular and Cellular Biochemistry on January 24th, 2022. See the published version at https://doi.org/10.1007/s11010-021-04337-5. 


\section{Abstract}

Matrix metalloproteinase (MMP) and soluble epoxide hydrolase (sEH) have completely unrelated biological functions, however, their dysregulation produce similar effects on biological systems. Based on the similarity in the reported structural requirements for their inhibition, the current study aimed to identify a simultaneous inhibitor for MMP and sEH. Six compounds were identified as potential simultaneous MMP/sEH inhibitors and tested for their capacity to inhibit MMP and sEH. Inhibition of MMP and sEH activity using their endogenous and exogenous substrates was measured by liquid chromatography/mass spectrometry, spectrophotometry and zymography. Two compounds, CTK8G1143 and ONO-4817, were identified to inhibit both MMP and SEH activity. CTK8G1143 and ONO-4817 inhibited the recombinant human sEH activity by an average of $67.4 \%$ and $55.2 \%$, respectively. $\mathrm{The}^{\mathrm{IC}} \mathrm{C}_{50}$ for CTK8G1143 and ONO-4817 to inhibit recombinant human sEH were 5.2 and $3.5 \mu \mathrm{M}$, respectively, whereas, their maximal inhibition values were $71.4 \%$ and $42.8 \%$, respectively. Also, MMP and sEH activity of human cardiomyocytes were simultaneously inhibited by CTK8G1143 and ONO-4817. Regarding other compounds, they showed either MMP or sEH inhibitory activity but not both. In conclusion, these two simultaneous inhibitors of MMP and sEH could provide a promising intervention for the prevention and control of several diseases, especially cardiovascular diseases.

\section{Introduction}

Matrix metalloproteinases (MMP) comprise a family of more than 20 zinc-dependent endopeptidases best known for their ability to mediate degradation of extracellular matrix (Jabłońska-Trypuć, Matejczyk, \& Rosochacki, 2016; Laronha \& Caldeira, 2020; X. Wang \& Khalil, 2018).. Extracellular matrix consists of a collection of fibrous proteins, collagen and enzymes imbedded in a hydrated polysaccharide gel, and provides the required structural and biochemical support to cells to form tissues and organs (Kular, Basu, \& Sharma, 2014; Laronha \& Caldeira, 2020). Therefore, degradation of extracellular matrix is the key step for tissue and organ remodeling either at normal physiological level, such as cell migration, proliferation and growth, or at pathological level, such as hypertrophy, carcinogenesis and metastasis (Azevedo, Prado, Antonio, Issa, \& Gerlach, 2014; Raeeszadeh-Sarmazdeh, Do, \& Hritz, 2020; X. Wang \& Khalil, 2018). On the other hand, soluble epoxide hydrolase (sEH) is the enzyme responsible for the inactivation of a group of important lipid mediators, namely epoxyeicosatrienoic acids (EETs) (Jorge H. Capdevila \& Wang, 2013; Xu et al., 2016). The formation of EETs is mediated by cytochrome P450 enzymes, typically CYP2Cs and CYP2Js, from arachidonic acid (J. H. Capdevila, Falck, \& Harris, 2000). The reported biological activities of EETs include anti-inflammatory, analgesic, vasodilatory, anti-platelet, antihypertrophic, fibrinolytic and vascular smooth muscle anti-migratory effects (Katakura et al., 2015; Christophe Morisseau \& Bruce D. Hammock, 2013; Yang et al., 2014).

Apparently, the independent inhibition of either MMP or sEH has several beneficial effects, therefore, they have been the focus of extensive biological research (Castro, Kandasamy, Youssef, \& Schulz, 2011; C. Morisseau \& B. D. Hammock, 2013). Despite the biological roles of MMP and sEH are completely unrelated, there is a remarkable agreement in the in vivo effects of their inhibitors, such as vasodilatory, 
anti-hypertrophic, anti-inflammatory and fibrinolytic effects (Imig, 2012; Vargova, Pytliak, \& Mechirova, 2012). Therefore, simultaneous inhibition of both MMP and SEH has great potential to yield synergistic and profound effect to control and prevent several diseases, especially cardiovascular diseases. Moreover, the simultaneous inhibition can attenuate adverse effects; there are rising concerns about the clinical use of sEH inhibitors due to their promoting effect on cancer progression (D. Wang \& Dubois, 2012). This major adverse effect can be controlled by the simultaneous inhibition of MMP, which is known to attenuate cancer progression and metastasis (Fields, 2019; Hu, Van den Steen, Sang, \& Opdenakker, 2007; Vargova et al., 2012). Interestingly, structure-activity relationship studies show several structural characteristics shared between number of MMP and sEH inhibitors (Levy et al., 1998; Morisseau et al., 1999; Morisseau et al., 2002; Pirard, 2007; Rezaee Zavareh et al., 2014; Shen \& Hammock, 2012; Tripathi et al., 2018). Accordingly, simultaneous inhibition of MMP and sEH by a single compound is feasible, therefore, in the current study, our objective was to identify a simultaneous inhibitor of MMP and SEH. We examined the chemical structure of several MMP and SEH inhibitors, consequently, 6 compounds (Table S1) were selected and tested for their capacity to simultaneously inhibit both MMP and $s E H$.

\section{Materials And Methods}

\section{Materials}

14,15-epoxyeicosatrienoic acid (14,15-EET), 14,15-dihydroxyeicosatrienoic acid (14,15-DHET), internal standards (14,15-EET-d11 and 14,15-DHET-d11), 4-nitrophenyl-2S,3S-epoxy-3 phenylpropyl carbonate (SNEPC) and 12-[[(tricyclo[3.3.1.13,7]dec-1-ylamino)carbonyl]amino]-dodecanoic acid (AUDA) were purchased from Cayman Chemical (Ann Arbor, MI). High performance liquid chromatography (HPLC) grade acetonitrile, water and ethyl acetate were purchased from EM Scientific (Gibbstawn, NJ). Acrylamide, $N^{\prime} N$ '-bis-methylene-acrylamide, $\beta$-mercaptoethanol, ammonium persulfate, glycine, and $\mathrm{N}, \mathrm{N}, \mathrm{N}^{\prime}, \mathrm{N}^{\prime}$-tetramethylethylenediamine (TEMED) were purchased from Bio-Rad Laboratories (Hercules, CA). 1-(1-methanesulfonyl-piperidin-4-yl)- 3-(4-trifluoromethoxy-phenyl)-urea (TUPS) was synthesized by Paul Jones (University of California, Davis) as described in Tsai et al (Tsai et al., 2010). Trans-4-[4-(3Adamantan-1-yl-ureido)-cyclohexyloxy]-benzoic acid (t-AUCB) was synthesized by Sung Hwang (University of California, Davis) as described in Hwang et al (Hwang, Tsai, Liu, Morisseau, \& Hammock, 2007). N-[((4,5-dihydro-5-thioxo-1,3,4-thiadiazol-2-yl)amino]carbonyl]-L-phenylalanine methyl ester (CTK8G1143) and N-[(1S,3S)-1-[(Ethoxymethoxy)methyl]-4-(hydroxyamino)-3-methyl-4-oxobutyl]-4phenoxybenzamide (ONO-4817) were purchased from Santa Cruz Biotechnology, Inc. (Santa Cruz, CA). 4aminophenyl mercuric acetate and doxycycline was purchased from Sigma-Aldrich Chemical Co. (St. Louis, MO). The catalytic domain of human recombinant MMP-2, human recombinant pro-MMP-2, human recombinant sEH and OmniMMP ${ }^{\circledR}$ fluorogenic peptide substrate were purchased from Oxford Biomedical Research (Oxford, MI). Other chemicals were purchased from Fisher Scientific Co. (Toronto, ON, Canada), or Sigma-Aldrich Chemical Co. (St. Louis, MO).

Determination of sEH activity by spectrophotometric assay 
The effect of MMP inhibitors on sEH activity was determined by using spectrophotometric substrate, SNEPC, as previously described by Dietze et al (Dietze, Kuwano, \& Hammock, 1994). The principle of assay is that the epoxide group in S-NEPC is hydrolysed by sEH, releasing 4-nitrophenol, which can be quantified spectrophotometrically. Briefly, 96-well plates were pretreated by $25 \mathrm{mg} / \mathrm{mL} \mathrm{BSA}, 2 \%$ Triton X-100 and $2 \%$ Tween 20 for $2 \mathrm{hr}$. Thereafter, $10 \mathrm{nM}$ of sEH was added to $0.1 \mathrm{mM}$ ethylenediaminetetraacetate and 2.5 $\mathrm{mg} / \mathrm{mL}$ of $\mathrm{BSA}$ in $76 \mathrm{mM}$ phosphate buffer $(\mathrm{pH}=6.4)$ in the absence or presence of $0.5 \mu \mathrm{M}$ of $\mathrm{sEH}$ inhibitor, t-AUCB, or $100 \mu \mathrm{M}$ of MMP inhibitors, CTK8G1143, ONO-4817 or doxycycline. After the initiation of the reaction by the addition of S-NEPC ( $30 \mu \mathrm{M}$ final concentration), test and blank (without SEH) samples were monitored at $405 \mathrm{~nm}$ every $10 \mathrm{sec}$ for $5 \mathrm{~min}$ at $25^{\circ} \mathrm{C}$ using BioTek Synergy $\mathrm{H} 1 \mathrm{Hybrid}$ Reader (BioTek Instruments, Inc., VT, USA). The initial rate of product formation in each well was determined by linear regression of absorbance-time data using GraphPad Prism (version 5.0, GraphPad software, San Diego, CA).

The effect of MMP inhibitors on sEH activity of immortalized human cardiomyocytes cell line, RL14, (American Type Culture Collection, Manassas, VA) was determined. Cells were maintained in Dulbecco's modified Eagle's medium F-12 medium supplemented with 12.5\% dialysed Fetal Bovine serum,and grown in $75 \mathrm{~cm}^{2}$ tissue culture flasks at $37^{\circ} \mathrm{C}$ in a $5 \% \mathrm{CO} 2$ humidified incubator. Cells were grown at a density of $5 \times 10^{5}$ cells per well in a 24-well tissue culture plate. On $\sim 80 \%$ confluence ( 2 days), the media was replaced by $300 \mu \mathrm{L}$ phosphate buffered saline in the absence or presence of $0.1 \mu \mathrm{M}$ of sEH inhibitor, tAUCB, or $10 \mu \mathrm{M}$ of MMP inhibitors, CTK8G1143 or ONO-4817. Thereafter, S-NEPC (30 $\mu \mathrm{M}$ final concentration) was added to the cells. After $10 \mathrm{~min}$ incubation at $37^{\circ} \mathrm{C}$, the formation of 4-nitrophenol in test and blank (without cells) samples were measured at $405 \mathrm{~nm}$ in 96-well plate using BioTek Synergy H1 Hybrid Reader (BioTek Instruments, Inc., VT, USA).

\section{Determination of sEH activity by liquid chromatography-mass spectrometry (LC-MS)}

The most important endogenous substrate of $\mathrm{sEH}$ is 14,15-EET, which is hydrolyzed by sEH to 14,15DHET. Liquid chromatography-electrospray ionization-mass spectrometry (LC-ESI-MS) (Waters Micromass ZQ 4000 spectrometer) was used to quantify the formation of 14,15-DHET as described previously by Morisseau and Hammock (Morisseau \& Hammock, 2007). Briefly, $5 \mathrm{nM}$ of sEH was added to $0.1 \mathrm{mg} / \mathrm{mL}$ BSA in $100 \mathrm{mM}$ phosphate buffer $(\mathrm{pH}=7.4)$ in the absence or presence of $0.5 \mu \mathrm{M}$ of sEH inhibitor, t-AUCB, or $100 \mu \mathrm{M}$ of MMP inhibitors, CTK8G1143, ONO-4817 or doxycycline. The reaction was initiated by the addition of 14,15-EET ( $50 \mu \mathrm{M}$ final concentration) and terminated after 7 min at $30^{\circ} \mathrm{C}$ by the addition of $600 \mathrm{ml}$ ice-cold acetonitrile followed by the internal standards. 14,15-EET and its corresponding 14,15-DHET in test and blank (without sEH) samples were extracted by $1 \mathrm{ml}$ ethyl acetate twice and dried using speed vacuum (Savant, Farmingdale, NY). Extracted 14,15-EET and 14,15-DHET were analyzed using LC-MS (Waters Micromass ZQ 4000 spectrometer) method as described previously (El-Sherbeni, Aboutabl, Zordoky, Anwar-Mohamed, \& El-Kadi, 2013). To determine the kinetics of sEH inhibition, concentration range of $0.05-500 \mathrm{nM}$ for TUPS or t-AUCB or $0.01-100 \mu \mathrm{M}$ for CTK8G1143 or 0.004-100 $\mu \mathrm{M}$ for ONO-4817 were tested using the same procedure as described above. Inhibitor 
concentrations required for $50 \%$ inhibition $\left(\mathrm{IC}_{50}\right)$ and maximal inhibition $\left(\mathrm{I}_{\max }\right)$ were determined by Enzyme Kinetics module from GraphPad Prism (version 5.0, GraphPad software, San Diego, CA).

\section{Determination of MMP-2 activity by fluorescence assay}

The effect of sEH inhibitors on MMP-2 activity was determined by assessing MMP-dependent hydrolysis of Mca-Pro-Leu-Gly-Leu-Dpa-Ala-Arg-NH2 AcOH (OmniMMP) fluorogenic substrate as previously described by Castro et al (Castro et al., 2013). 96-well black polystyrene half-area plates were pretreated by $25 \mathrm{mg} / \mathrm{mL}$ BSA, $2 \%$ Triton X-100 and $2 \%$ Tween 20 for $2 \mathrm{hr}$. Thereafter, $0.5 \mathrm{nM}$ of MMP-2 catalytic domain was added to $10 \mathrm{mM} \mathrm{CaCl} 2,0.05 \%$ Brij 35 and $10 \mathrm{mM} \mathrm{ZnSO} 4$ in $50 \mathrm{mM}$ Tris- $\mathrm{HCl}(\mathrm{pH}=7.0)$ in the absence or presence of $100 \mu \mathrm{M}$ of MMP inhibitor, ONO-4817, or $50 \mu \mathrm{M}$ of sEH inhibitors, AUDA, TUPS or tAUCB. The reaction was initiated by the addition of OmniMMP substrate (30 $\mu \mathrm{M}$ final concentration). The fluorescence associated with the breakdown of OmniMMP in test and blank (without MMP-2) samples were measured at $30 \mathrm{~s}$ intervals (excitation and emission wavelengths of $328 \mathrm{~nm}$ and $393 \mathrm{~nm}$ ) for $1 \mathrm{hr}$ at $37^{\circ} \mathrm{C}$ using a BioTek Synergy H1 Hybrid Reader (BioTek Instruments, Inc., VT, USA). The initial rate of product formation in each well was determined by linear regression of fluorescence-time data using GraphPad Prism (version 5.0, GraphPad software, San Diego, CA).

\section{Determination of MMP activity by zymography}

The effect of sEH inhibitors on the gelatinolytic activity of MMP was also determined using substrate-gel electrophoresis (zymography) as previously described by Hawkes et al (Hawkes, Li, \& Taniguchi, 2010). Briefly, pro-MMP-2 was activated by $1 \mathrm{mM}$ 4-aminophenyl mercuric acetate in $150 \mathrm{mM} \mathrm{NaCl}, 5 \mathrm{mM} \mathrm{CaCl}$, $50 \mathrm{mM}$ Tris $\mathrm{HCl}(\mathrm{pH}=7.5)$. Then, pro-MMP-2 and MMP-2 were electrophoresed on an $8 \%$ polyacrylamide gel containing $1 \mathrm{mg} / \mathrm{ml}$ gelatin for $90 \mathrm{~min}$ ( $125 \mathrm{~V}$, ambient temperature). Thereafter, the gels were cut into individual strips and then washed in $2.5 \%$ Triton X-100 and $5 \mathrm{mM} \mathrm{CaCl} 2$ in $50 \mathrm{mM}$ Tris-HCl buffer $(\mathrm{pH}=8.0)$. These strips were incubated separately overnight at $37^{\circ} \mathrm{C}$ in development buffer $(50 \mathrm{mM}$ Tris$\mathrm{HCl}, 10 \mathrm{mM} \mathrm{CaCl}_{2}, 1 \mu \mathrm{M} \mathrm{ZnSO}_{4}, \mathrm{pH}^{7.6}$ ) in the absence or presence of $100 \mu \mathrm{M}$ of MMP inhibitor, ONO4817 , or $50 \mu \mathrm{M}$ of sEH inhibitors, AUDA, TUPS or t-AUCB. Gels were stained with $1 \mathrm{mg} / \mathrm{mL}$ Coomassie blue, followed by destaining and, finally, gelatinolytic activity appeared as a clear band on a blue background. Moreover, gelatinolytic activity of MMP-2 and MMP-9 in the conditioned medium of cultured RL14 was determined by zymography, where $8 \mu \mathrm{L}$ aliquots of conditioned RL14 medium were electrophoresed as described above. After washing, gel strips were incubated separately overnight at $37^{\circ} \mathrm{C}$ in development buffer, as described above, in the absence or presence of $10 \mu \mathrm{M}$ CTK8G1143 or ONO4817.

\section{Results}

\section{The effect of MMP inhibitors on sEH activity}

The chemical structure of several MMP inhibitors were reviewed, and consequently, 2 MMP inhibitors, CTK8G1143 and ONO-4817, were selected based on the presence of sEH inhibitors pharmacophores as 
previously reported (Morisseau et al., 1999, Morisseau et al., 2002, Ulu et al., 2012). In addition, we included doxycycline, which exerts its MMP inhibitory activity through the same mechanism of CTK8G1143 and ONO-4817, chelating the catalytic metal $\left(\mathrm{Zn}^{2+}\right)$ ions, albeit having totally different chemical structure (Table S1) (Castro et al., 2011). Using S-NEPC as a spectrophotometric exogenous substrate, CTK8G1143 and ONO-4817 were found to exhibit inhibitory activity against recombinant human sEH enzyme (Fig. 1A). CTK8G1143 and ONO-4817 inhibited sEH activity by $59.8 \%$ and $71.2 \%$, respectively, whereas the potent sEH inhibitor, t-AUCB, inhibited sEH activity by $85.5 \%$ compared with dimethyl sulfoxide (DMSO) control (Fig. 1A). Using 14,15-EET, which is the endogenous substrate of sEH, CTK8G1143 and ONO-4817 inhibited sEH activity by $74.9 \%$ and $39.1 \%$, respectively, and t-AUCB inhibited sEH activity by $84.2 \%$ compared with DMSO control (Fig. $1 \mathrm{~A}$ ). In both cases, doxycycline did not significantly affect the activity of sEH compared with DMSO control (Fig. 1A). The effect of sEH inhibitors on MMP-2 activity

In contrast to MMP inhibitors, sEH inhibitors display a more homogenous group with respect to chemical structure, from which we selected 3 potent sEH inhibitors, AUDA, TUPS and t-AUCB. The selected sEH inhibitors show some general structural characteristics of MMP inhibitors, most importantly Zn-binding moieties, such as carboxyl and sulfonyl groups (Table S1) (Fisher \& Mobashery, 2006, Hu et al., 2007, Scozzafava \& Supuran, 2000). Either by using fluorogenic substrate or zymography, AUDA, TUPS or tAUCB did not cause any significant alteration in MMP-2 activity compared with DMSO control (Fig. 1B). On the other hand, ONO-4817 inhibited MMP-2 activity by $98.3 \%$ and $99.4 \%$ using fluorescence assay and zymography, respectively (Fig. 1B).

\section{The determination of sEH inhibition kinetics of MMP inhibitors}

In order to determine whether the inhibitory effect of MMP inhibitors on sEH can occur at biologicallyrelevant concentrations of MMP inhibitors, we determined SEH inhibition kinetics of CTK8G1143 and ONO-4817 by LC-MS. The maximal inhibition ( $\left.I_{\max }\right)$ of sEH activity by CTK8G1143 and ONO-4817 were $71.4 \%$ and $42.8 \%$, respectively, compared with DMSO control (Fig. 2 \& Table 1). The IC 50 values were $5.2 \mu \mathrm{M}$ and $3.5 \mu \mathrm{M}$ for CTK8G1143 and ONO-4817, respectively (Fig. 2 \& Table 1). The IC 50 values for the potent sEH inhibitors, TUPS and t-AUCB, were $6.5 \mathrm{nM}$ and $12.3 \mathrm{nM}$ and $I_{\max }$ values were $60.7 \%$ and $84.5 \%$, respectively (Fig. $2 \&$ Tab.1). Despite MMP inhibitors were remarkably less potent than sEH inhibitors with respect to $s E H$ inhibition, the concentration range of sEH inhibition by MMP inhibitors is within the reported plasma concentration range (Ro et al., 2007, Shiraga et al., 2002).

\section{The effect of MMP inhibitors on sEH and MMP activities in RL14 cells}

MMP enzymes are mostly secreted proteins, in contrast to $\mathrm{sEH}$, which is located in cell cytoplasm. Therefore, we examined whether MMP inhibitors can freely penetrate cell membrane in order to simultaneously inhibit MMP outside the cell and sEH inside the cell at a biologicallyrelevant concentration. Exposing RL14 cells to $10 \mu \mathrm{M}$ of CTK8G1143 or ONO-4817 significantly inhibited the intracellular sEH activity by $63.3 \%$ and $34.5 \%$, respectively, whereas, t-AUCB inhibited sEH activity by 
86.4\% compared with DMSO control (Fig. 3A). Also, $10 \mu \mathrm{M}$ of CTK8G1143 and ONO-4817 inhibited the extracellular MMP-2 activity by $52.2 \%$ and $61.3 \%$ and MMP-9 activity by $57.7 \%$ and $71.6 \%$, respectively, compared with DMSO control (Fig. 3B).

\section{Discussion}

Following the discovery of the important biological roles of MMP and $\mathrm{sEH}$, great efforts have been made to design potent inhibitors for them. MMP comprise several enzymes, which depend on zinc for their activity. Zinc as a divalent ion is bound to a common catalytic domain shared between all MMP. Therefore, the most important feature for MMP inhibitor is the presence of zinc-binding group, besides the proper positioning in the catalytic site by non-covalent interactions. Aside of these features, MMP inhibitors are remarkably diverse and are usually subdivided into 8 classes, succinyl hydroxamates, sulphonamide hydroxamates, phosphinamide hydroxamates, carboxylates, thiol inhibitors, aminomethyl benzimidazole analogue, peptides and tetracyclines. In contrast, sEH inhibitors are structurally similar, Morisseau et a/ first reported that carbamide compounds with appropriate substituents can exhibit potent sEH inhibitory activity (Morisseau et al., 1999). Till now, the carbamide pharmacophore presents in all current $\mathrm{SEH}$ inhibitors that can be divided into urea, benzamide and benzoxazole compounds (Chen, Zhang, Li, \& Han, 2012, C. Morisseau \& B. D. Hammock, 2013, Shen \& Hammock, 2012).

Due to the diversity of MMP inhibitors, we scanned MMP inhibitors for urea, benzamide and benzoxazole moiety. We did not find any MMP inhibitor with benzoxazole moiety, on the other hand, urea moiety was found in CTK8G1143, and benzamide moiety was found in ONO-4817. In addition to MMP-2 and MMP-3 inhibitory activity of CTK8G1143, it possesses several structural characteristics critical for a successful urea-based sEH inhibitor (Table S1). The sEH inhibition structural characteristics of CTK8G1143 include: 1) urea group, 2) each of the two urea nitrogen atoms is singly substituted by a bulky group (6-12 carbons), 3) the urea $\mathrm{N}$-substituents are of unequal size, and 4) a secondary pharmacophore, an ester or ether group, present at a distance from urea carbonyl group, which reported to improve the effectiveness of sEH inhibitors (Huang et al., 2010, Kim et al., 2005, Morisseau et al., 1999, Morisseau et al., 2002, Shen \& Hammock, 2012). In addition, ONO-4817, which is a broad-spectrum MMP inhibitor, shows several structural characteristics of a benzamide-based sEH inhibitor (Table S1). This includes benzamide moiety with an electronegative function group in the para-position and $\mathrm{N}$-attached to a very bulky group (12-15 carbons) ended with methylsulfonyl or carboxyl group (Eldrup et al., 2009, C. Morisseau \& B. D. Hammock, 2013, Rezaee Zavareh et al., 2014). We were also interested in measuring the MMP inhibitory activity of 3 urea-based sEH inhibitors, AUDA, TUPS and t-AUCB, due to their structural similarity with CTK8G114 (Table S1). In the current study, we examined these 5 compounds and determined CTK8G114 and ONO-4817 as simultaneous inhibitors of MMP and sEH. We can conclude that sEH inhibitory activity of CTK8G114 and ONO-4817 cannot be attributed to zinc-binding activity of these MMP inhibitors based on doxycycline lackingof sEH inhibitory activity. With respect to AUDA, TUPS and t-AUCB, they did not inhibit MMP-2 activity, despite their potent inhibitory effect on sEH. This can be explained by their manyfold less potent zinc-binding groups, carboxyl and methylsulfonyl. Typically, MMP inhibitors have 
hydroxamate as zinc-binding group, which has been reported to be 20 -fold to 50 -fold more potent than thiol group and 100-2000 folds more potent than carboxyl group (Hu et al., 2007).

Over 20 years ago, there was great enthusiasm for MMP inhibitors as anticancer agents by impairing tumor angiogenesis and metastasis. However, disappointing results from multiple cancer clinical trials have reduced the interest in MMP inhibitors (Vandenbroucke \& Libert, 2014, Vargova et al., 2012). In fact, elevated MMP activity has been reported not only in malignant diseases but also in several inflammatory and degenerative cardiovascular diseases (Cabral-Pacheco et al., 2020). The maladaptive remodeling mediated by MMP is an important aspect in cardiac hypertrophy, heart failure, hypertension, and atherosclerosis, therefore, MMP inhibition could be beneficial for these diseases. Similarly, hypertension and several inflammatory diseases were once regarded the prime indications for the use of sEH inhibitors. However, sEH inhibitors failed in clinical trials to show promising results and the only sEH inhibitor in clinical trial now is GSK2256294 that has entered Phase I clinical trial for a chronic obstructive pulmonary disease in male obese smokers (Podolin et al., 2013). Consequently, simultaneous MMP/sEH inhibition could be a promising solution to potentiate the benefits of the individual MMP or SEH inhibition. In conclusion, we identified 2 compounds that exhibit simultaneous inhibitory activity against MMP and $\mathrm{sEH}$ at biologically relevant feasible concentration range. Further investigation is required to evaluate their use for the prevention and control of several diseases, especially cardiovascular diseases.

\section{Declarations}

\section{Funding}

This work was supported by a grant from the Canadian Institutes of Health Research (CIHR) [Grant MOP 106665]. RB is the recipient of Alberta Innovates-Health Solutions summer studentship.

\section{Author information}

\section{Affiliations}

${ }^{1}$ Faculty of Pharmacy and Pharmaceutical Sciences, University of Alberta, Edmonton, Alberta, Canada T6G 2E1

${ }^{2}$ Department of Clinical Pharmacy, Faculty of Pharmacy, Tanta University, Tanta, Egypt

Ahmed A. El-Sherbeni ${ }^{2}$, Rabia Bhatti ${ }^{1}$, Fadumo A. Isse ${ }^{1}$, Ayman O.S. El-Kadi ${ }^{1}$

\section{Contributions}

Ahmed A. El-Sherbeni: Performing experiments, data analysis, writing the manuscript

Rabia Bhatti: Performing some experiments

Fadumo A. Isse: data analysis, writing the manuscript 
Ayman O.S. El-Kadi: data analysis, writing the manuscript

\section{Corresponding author}

Correspondence to Ayman O.S. El-Kadi

\section{Data availability}

The datasets used or analyzed during the current study are available from the corresponding author on reasonable request.

\section{Ethical approval}

No animals were used for these studies.

\section{Conflict of Interest}

The research was conducted in the absence of any commercial or financial relationships that could be construed as a potential conflict of interest.

\section{References}

1. Azevedo, A., Prado, A. F., Antonio, R. C., Issa, J. P., \& Gerlach, R. F. (2014). Matrix metalloproteinases are involved in cardiovascular diseases. Basic Clin Pharmacol Toxicol, 115(4), 301-314. doi:10.1111/bcpt.12282

2. Cabral-Pacheco, G. A., Garza-Veloz, I., Castruita-De la Rosa, C., Ramirez-Acuña, J. M., Perez-Romero, B. A., Guerrero-Rodriguez, J. F., . . Martinez-Fierro, M. L. (2020). The Roles of Matrix Metalloproteinases and Their Inhibitors in Human Diseases. International journal of molecular sciences, 21(24), 9739. doi:10.3390/ijms21249739

3. Capdevila, J. H., Falck, J. R., \& Harris, R. C. (2000). Cytochrome P450 and arachidonic acid bioactivation. Molecular and functional properties of the arachidonate monooxygenase. $J$ Lipid Res, 41(2), 163-181. Retrieved from http://www.ncbi.nlm.nih.gov/pubmed/10681399

4. Capdevila, J. H., \& Wang, W. (2013). Role of cytochrome P450 epoxygenase in regulating renal membrane transport and hypertension. Current Opinion in Nephrology and Hypertension, 22, 16S 169.

5. Castro, M. M., Fuah, J., Ali, M., Sung, M., Schulz, J., Kondo, M. Y., . . Schulz, R. (2013). Inhibitory effects of caspase inhibitors on the activity of matrix metalloproteinase-2. Biochem Pharmacol, 86(4), 469-475. doi:10.1016/j.bcp.2013.06.003

6. Castro, M. M., Kandasamy, A. D., Youssef, N., \& Schulz, R. (2011). Matrix metalloproteinase inhibitor properties of tetracyclines: therapeutic potential in cardiovascular diseases. Pharmacol Res, 64(6), 551-560. doi:10.1016/j.phrs.2011.05.005 
7. Chen, H., Zhang, Y., Li, L., \& Han, J. G. (2012). Probing ligand-binding modes and binding mechanisms of benzoxazole-based amide inhibitors with soluble epoxide hydrolase by molecular docking and molecular dynamics simulation. J Phys Chem B, 116(34), 10219-10233. doi:10.1021/jp304736e

8. Dietze, E. C., Kuwano, E., \& Hammock, B. D. (1994). Spectrophotometric substrates for cytosolic epoxide hydrolase. Anal Biochem, 216(1), 176-187. doi:10.1006/abio.1994.1023

9. El-Sherbeni, A. A., Aboutabl, M. E., Zordoky, B. N., Anwar-Mohamed, A., \& El-Kadi, A. O. (2013). Determination of the dominant arachidonic acid cytochrome p450 monooxygenases in rat heart, lung, kidney, and liver: protein expression and metabolite kinetics. AAPS J, 15(1), 112-122. doi:10.1208/s12248-012-9425-7

10. Eldrup, A. B., Soleymanzadeh, F., Taylor, S. J., Muegge, I., Farrow, N. A., Joseph, D., . . De Lombaert, S. (2009). Structure-based optimization of arylamides as inhibitors of soluble epoxide hydrolase. J Med Chem, 52(19), 5880-5895. doi:10.1021/jm9005302

11. Fields, G. B. (2019). Mechanisms of Action of Novel Drugs Targeting Angiogenesis-Promoting Matrix Metalloproteinases. Frontiers in Immunology, 10(1278). doi:10.3389/fimmu.2019.01278

12. Fisher, J. F., \& Mobashery, S. (2006). Recent advances in MMP inhibitor design. Cancer Metastasis Rev, 25(1), 115-136. doi:10.1007/s10555-006-7894-9

13. Hawkes, S. P., Li, H., \& Taniguchi, G. T. (2010). Zymography and reverse zymography for detecting MMPs and TIMPs. Methods Mol Biol, 622, 257-269. doi:10.1007/978-1-60327-299-5_16

14. Hu, J., Van den Steen, P. E., Sang, Q. X., \& Opdenakker, G. (2007). Matrix metalloproteinase inhibitors as therapy for inflammatory and vascular diseases. Nat Rev Drug Discov, 6(6), 480-498. doi: $10.1038 / \mathrm{nrd} 2308$

15. Huang, S.-X., Li, H.-Y., Liu, J.-Y., Morisseau, C., Hammock, B. D., \& Long, Y.-Q. (2010). Incorporation of Piperazino Functionality into 1,3-Disubstituted Urea as the Tertiary Pharmacophore Affording Potent Inhibitors of Soluble Epoxide Hydrolase with Improved Pharmacokinetic Properties. Journal of medicinal chemistry, 53(23), 8376-8386. doi:10.1021/jm101087u

16. Hwang, S. H., Tsai, H. J., Liu, J. Y., Morisseau, C., \& Hammock, B. D. (2007). Orally bioavailable potent soluble epoxide hydrolase inhibitors. J Med Chem, 50(16), 3825-3840. doi:10.1021/jm070270t

17. Imig, J. D. (2012). Epoxides and soluble epoxide hydrolase in cardiovascular physiology. Physiol Rev, 92(1), 101-130. doi:10.1152/physrev.00021.2011

18. /1/101 [pii]

19. Jabłońska-Trypuć, A., Matejczyk, M., \& Rosochacki, S. (2016). Matrix metalloproteinases (MMPs), the main extracellular matrix (ECM) enzymes in collagen degradation, as a target for anticancer drugs. $J$ Enzyme Inhib Med Chem, 31(sup1), 177-183. doi:10.3109/14756366.2016.1161620

20. Katakura, M., Hashimoto, M., Inoue, T., Mamun, A. A., Tanabe, Y., Arita, M., \& Shido, O. (2015). Chronic Arachidonic Acid Administration Decreases Docosahexaenoic Acid- and Eicosapentaenoic AcidDerived Metabolites in Kidneys of Aged Rats. PLoS One, 10(10), e0140884-e0140884. doi:10.1371/journal.pone.0140884 
21. Kim, I. H., Heirtzler, F. R., Morisseau, C., Nishi, K., Tsai, H. J., \& Hammock, B. D. (2005). Optimization of amide-based inhibitors of soluble epoxide hydrolase with improved water solubility. J Med Chem, 48(10), 3621-3629. doi:10.1021/jm0500929

22. Kular, J. K., Basu, S., \& Sharma, R. I. (2014). The extracellular matrix: Structure, composition, agerelated differences, tools for analysis and applications for tissue engineering. $J$ Tissue Eng, 5, 2041731414557112. doi:10.1177/2041731414557112

23. Laronha, H., \& Caldeira, J. (2020). Structure and Function of Human Matrix Metalloproteinases. Cells, 9(5), 1076. doi:10.3390/cells9051076

24. Levy, D. E., Lapierre, F., Liang, W., Ye, W., Lange, C. W., Li, X., . . Galardy, R. E. (1998). Matrix metalloproteinase inhibitors: a structure-activity study. J Med Chem, 41(2), 199-223. doi:10.1021/jm970494j

25. Morisseau, C., Goodrow, M. H., Dowdy, D., Zheng, J., Greene, J. F., Sanborn, J. R., \& Hammock, B. D. (1999). Potent urea and carbamate inhibitors of soluble epoxide hydrolases. Proc Natl Acad Sci U S A, 96(16), 8849-8854. Retrieved from http://www.ncbi.nlm.nih.gov/pubmed/10430859

26. Morisseau, C., Goodrow, M. H., Newman, J. W., Wheelock, C. E., Dowdy, D. L., \& Hammock, B. D. (2002). Structural refinement of inhibitors of urea-based soluble epoxide hydrolases. Biochem Pharmacol, 63(9), 1599-1608. Retrieved from http://www.ncbi.nlm.nih.gov/pubmed/12007563

27. Morisseau, C., \& Hammock, B. D. (2007). Measurement of soluble epoxide hydrolase (sEH) activity. Curr Protoc Toxicol, Chapter 4, Unit 4 23. doi:10.1002/0471140856.tx0423s33

28. Morisseau, C., \& Hammock, B. D. (2013). Impact of soluble epoxide hydrolase and epoxyeicosanoids on human health. Annu Rev Pharmacol Toxicol, 53, 37-58. doi:10.1146/annurev-pharmtox-011112140244

29. Morisseau, C., \& Hammock, B. D. (2013). Impact of soluble epoxide hydrolase and epoxyeicosanoids on human health. Annual review of pharmacology and toxicology, 53, 37-58. doi:10.1146/annurevpharmtox-011112-140244

30. Pirard, B. (2007). Insight into the structural determinants for selective inhibition of matrix metalloproteinases. Drug Discov Today, 12(15-16), 640-646. doi:10.1016/j.drudis.2007.06.003

31. Podolin, P. L., Bolognese, B. J., Foley, J. F., Long, E., 3rd, Peck, B., Umbrecht, S., . . Callahan, J. F. (2013). In vitro and in vivo characterization of a novel soluble epoxide hydrolase inhibitor. Prostaglandins Other Lipid Mediat, 104-105, 25-31. doi:10.1016/j.prostaglandins.2013.02.001

32. $\mathrm{S} 1098-8823(13) 00007-5$ [pii]

33. Raeeszadeh-Sarmazdeh, M., Do, L. D., \& Hritz, B. G. (2020). Metalloproteinases and Their Inhibitors: Potential for the Development of New Therapeutics. Cells, 9(5). doi:10.3390/cells9051313

34. Rezaee Zavareh, E., Hedayati, M., Hoghooghi Rad, L., Shahhosseini, S., Faizi, M., \& Tabatabai, S. A. (2014). Design, synthesis and biological evaluation of 4-benzamidobenzoic Acid hydrazide derivatives as novel soluble epoxide hydrolase inhibitors. Iran J Pharm Res, 13(Suppl), 51-59. Retrieved from http://www.ncbi.nlm.nih.gov/pubmed/24711829 
35. Ro, Y., Hamada, C., Inaba, M., lo, H., Kaneko, K., \& Tomino, Y. (2007). Inhibitory effects of matrix metalloproteinase inhibitor ONO-4817 on morphological alterations in chlorhexidine gluconateinduced peritoneal sclerosis rats. Nephrol Dial Transplant, 22(10), 2838-2848.

doi:10.1093/ndt/gfm323

36. Scozzafava, A., \& Supuran, C. T. (2000). Carbonic anhydrase and matrix metalloproteinase inhibitors: sulfonylated amino acid hydroxamates with MMP inhibitory properties act as efficient inhibitors of CA isozymes I, II, and IV, and N-hydroxysulfonamides inhibit both these zinc enzymes. J Med Chem, 43(20), 3677-3687. Retrieved from http://www.ncbi.nlm.nih.gov/pubmed/11020282

37. Shen, H. C., \& Hammock, B. D. (2012). Discovery of Inhibitors of Soluble Epoxide Hydrolase: A Target with Multiple Potential Therapeutic Indications. Journal of medicinal chemistry, 55(5), 1789-1808. doi:10.1021/jm201468j

38. Shiraga, M., Yano, S., Yamamoto, A., Ogawa, H., Goto, H., Miki, T., .. Sone, S. (2002). Organ heterogeneity of host-derived matrix metalloproteinase expression and its involvement in multipleorgan metastasis by lung cancer cell lines. Cancer Res, 62(20), 5967-5973. Retrieved from http://www.ncbi.nlm.nih.gov/pubmed/12384564

39. Tripathi, N., Paliwal, S., Sharma, S., Verma, K., Gururani, R., Tiwari, A., . . Pant, A. (2018). Discovery of Novel Soluble Epoxide Hydrolase Inhibitors as Potent Vasodilators. Scientific Reports, 8(1), 14604. doi:10.1038/s41598-018-32449-4

40. Tsai, H. J., Hwang, S. H., Morisseau, C., Yang, J., Jones, P. D., Kasagami, T., ... Hammock, B. D. (2010). Pharmacokinetic screening of soluble epoxide hydrolase inhibitors in dogs. Eur J Pharm Sci, 4O(3), 222-238. doi:10.1016/j.ejps.2010.03.018

41. S0928-0987(10)00101-6 [pii]

42. Ulu, A., Appt, S., Morisseau, C., Hwang, S. H., Jones, P. D., Rose, T. E., . . Hammock, B. D. (2012). Pharmacokinetics and in vivo potency of soluble epoxide hydrolase inhibitors in cynomolgus monkeys. Br J Pharmacol, 165(5), 1401-1412. doi:10.1111/j.1476-5381.2011.01641.x

43. Vandenbroucke, R. E., \& Libert, C. (2014). Is there new hope for therapeutic matrix metalloproteinase inhibition? Nature Reviews Drug Discovery, 13(12), 904-927. doi:10.1038/nrd4390

44. Vargova, V., Pytliak, M., \& Mechirova, V. (2012). Matrix metalloproteinases. EXS, 103, 1-33. doi:10.1007/978-3-0348-0364-9_1

45. Wang, D., \& Dubois, R. N. (2012). Epoxyeicosatrienoic acids: a double-edged sword in cardiovascular diseases and cancer. J Clin Invest, 122(1), 19-22. doi:10.1172/JCl61453

46. Wang, X., \& Khalil, R. A. (2018). Matrix Metalloproteinases, Vascular Remodeling, and Vascular Disease. Adv Pharmacol, 81, 241-330. doi:10.1016/bs.apha.2017.08.002

47. Xu, X., Li, R., Chen, G., Hoopes, S. L., Zeldin, D. C., \& Wang, D. W. (2016). The Role of Cytochrome P450 Epoxygenases, Soluble Epoxide Hydrolase, and Epoxyeicosatrienoic Acids in Metabolic Diseases. Adv Nutr, 7(6), 1122-1128. doi:10.3945/an.116.012245

48. Yang, C., Yang, J., Xu, X., Yan, S., Pan, S., Pan, X., . . Leung, G. P. (2014). Vasodilatory effect of 14,15epoxyeicosatrienoic acid on mesenteric arteries in hypertensive and aged rats. Prostaglandins Other 


\section{Tables}

Table 1. Inhibitor concentration required for $50 \%$ inhibition $\left(\mathrm{IC}_{50}\right)$ and maximal inhibition $\left(\mathrm{I}_{\max }\right)$ mean values and SE.

\begin{tabular}{llll} 
& $\mathbf{I C}_{\mathbf{5 0}} \pm \mathbf{S E}$ & $\mathrm{I}_{\max } \pm \mathrm{SE}$ & $\mathbf{R}^{2}$ \\
\hline TUPS & $0.01 \pm 0.00$ & $60.73 \pm 5.78$ & 0.95 \\
\hline tAUCB & $0.01 \pm 0.00$ & $84.53 \pm 5.64$ & 0.93 \\
\hline CTK8G1143 & $5.20 \pm 0.19$ & $71.39 \pm 2.85$ & 0.89 \\
\hline ONO-4817 & $3.48 \pm 0.12$ & $42.82 \pm 7.28$ & 0.88 \\
\hline $\mathrm{IC}_{50}$ is in $\mu \mathrm{M}$, and $\mathrm{I}_{\max }$ is \% of DMSO control. &
\end{tabular}

Figures

FIGURE 1

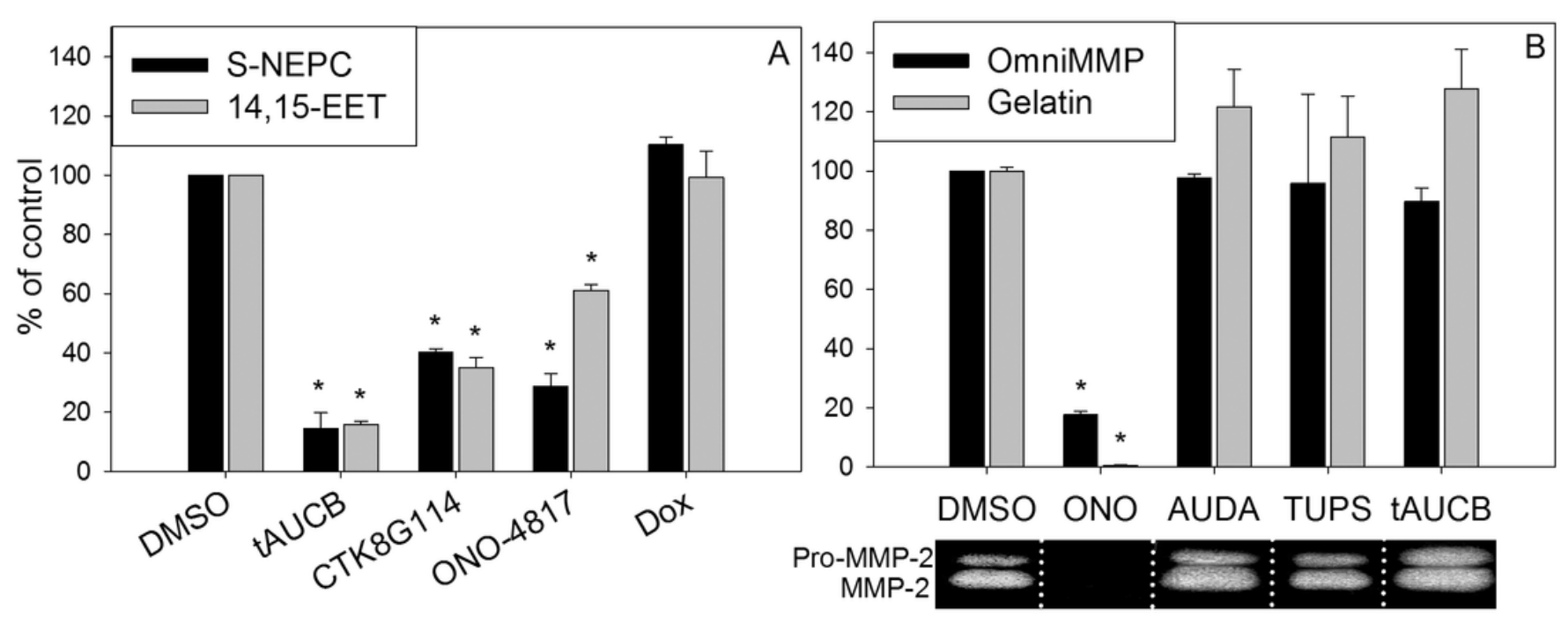

Figure 1 
Effect of CTK8G114, ONO-4817, doxycycline, AUDA, TUPS and tAUCB on MMP-2 and sEH activities. (A) the activity of human recombinant $\mathrm{SEH}$ was measured by using exogenous substrate, S-NEPC, and by using sEH endogenous substrate, 14,15-EET, as described under Materials and Methods in the absence or presence of $0.5 \mu \mathrm{M}$ of sEH inhibitor, t-AUCB, or $100 \mu \mathrm{M}$ of MMP inhibitors, CTK8G1143, ONO-4817 or doxycycline. (B) the activity of human recombinant MMP-2 catalytic domain or the activated human recombinant Pro-MMP-2 was measured by using exogenous substrate, OmniMMP, and by using MMP endogenous substrate, gelatin, as described under Materials and Methods in the absence or presence of $100 \mu \mathrm{M}$ of MMP inhibitor, ONO-4817, or $50 \mu \mathrm{M}$ sEH inhibitors, AUDA, TUPS or t-AUCB. Results are presented as mean \% of DMSO control and SE, and are based on 4 individual experiments. ${ }^{*} p<0.05$.

FIGURE 2

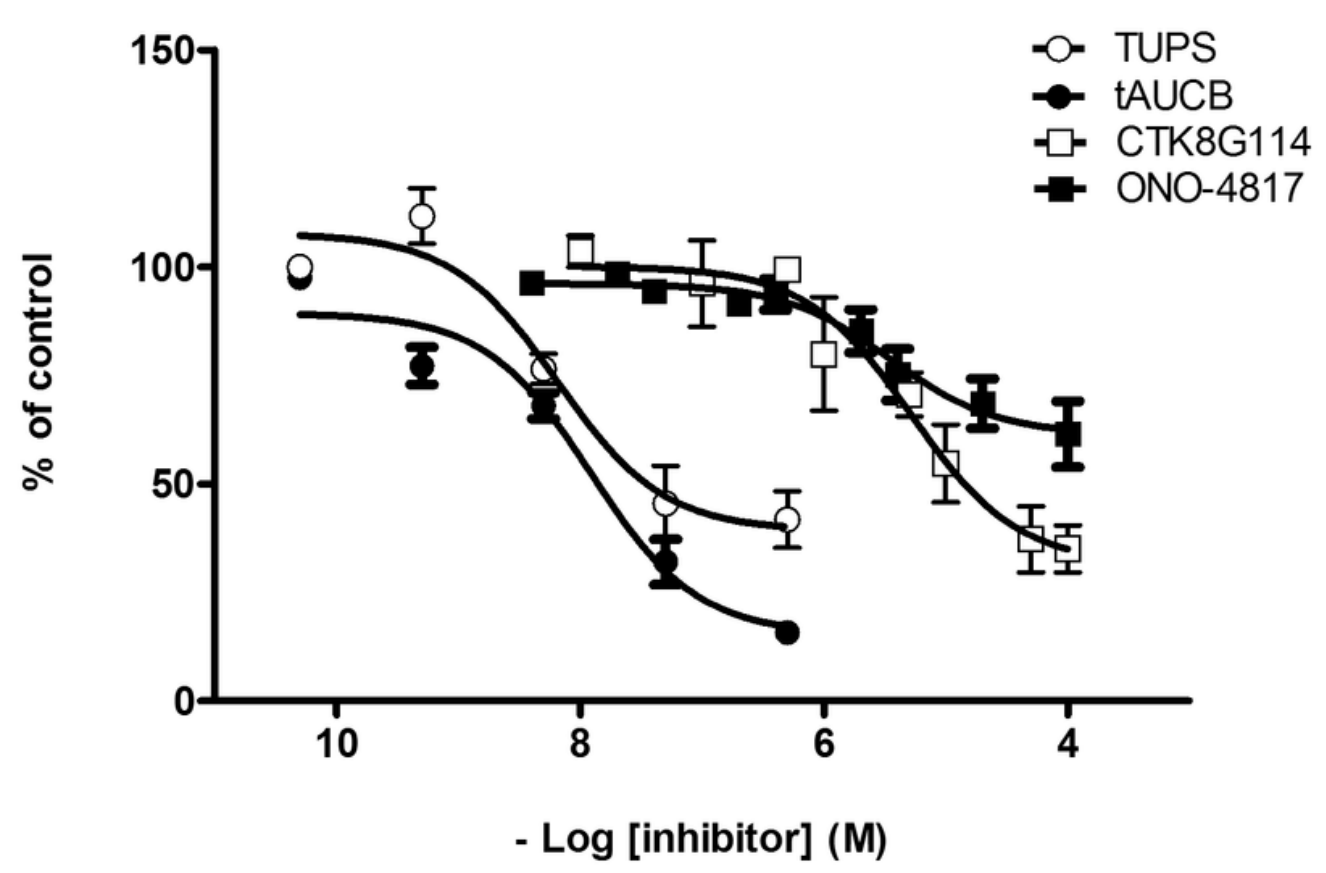

Figure 2

sEH inhibition kinetics of CTK8G114, ONO-4817, TUPS and tAUCB. Concentration range of 0.05-500 nM for TUPS or t-AUCB or 0.01-100 $\mu \mathrm{M}$ for CTK8G1143 or 0.004-100 $\mu \mathrm{M}$ for ONO-4817 were incubated with human recombinant $s E H$. Activity was measured by the formation of 14,15-DHET from 14,15-EET, as described under Materials and Methods. Results are presented as mean \% of DMSO control and SE, and are based on 4 individual experiments. 
FIGURE 3

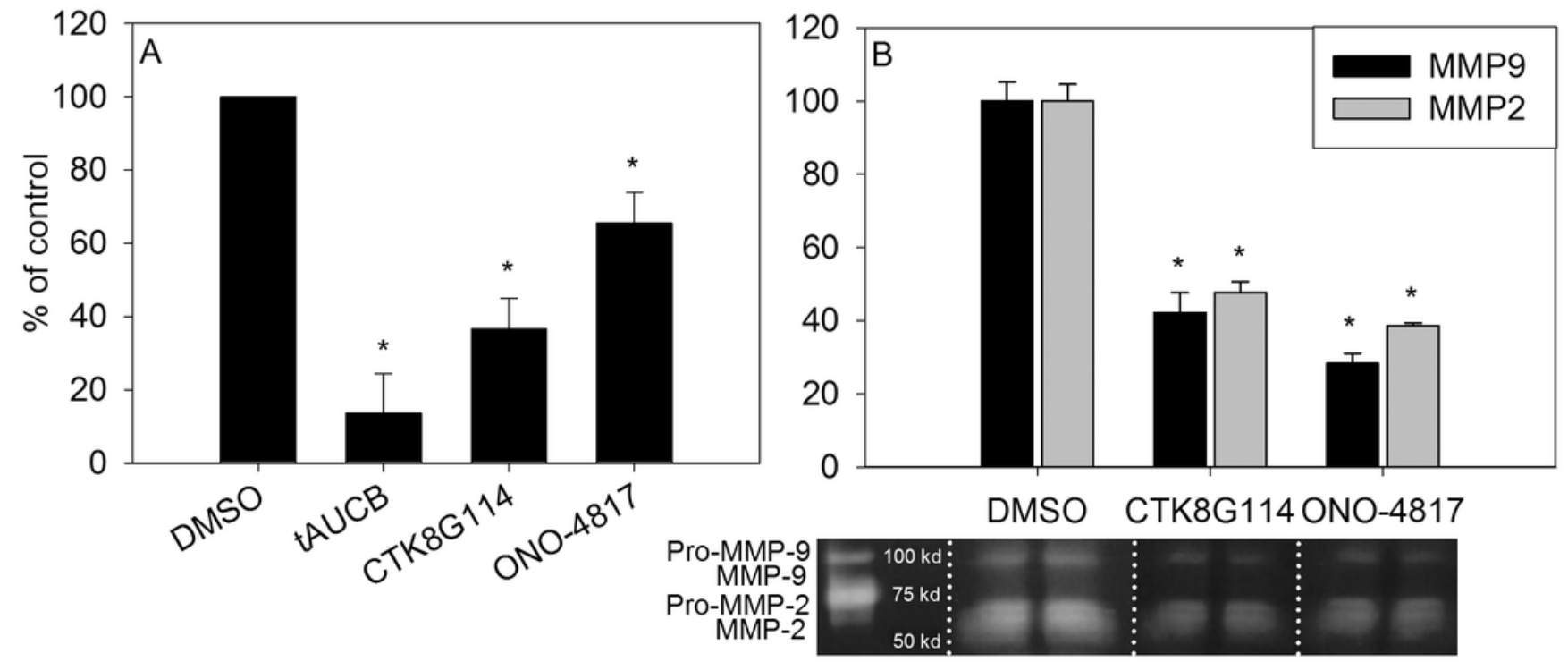

Figure 3

Effect of CTK8G114, ONO-4817 and tAUCB on MMP and sEH activities in human cardiomyocytes, RL14. These compounds were incubated with RL14 cells or conditioned medium of cultured RL14. SEH activity in the absence or presence of $0.1 \mu \mathrm{M}$ of sEH inhibitor, t-AUCB, or $10 \mu \mathrm{M}$ of MMP inhibitors, CTK8G1143 or ONO-4817 (A), as well as MMP-2 and MMP-9 activities in the absence or presence of $10 \mu \mathrm{M}$ CTK8G1143 or ONO-4817 (B) were measured as described under Materials and Methods. Results are presented as mean \% of DMSO control and SE, and are based on 4 individual experiments. ${ }^{*} p<0.05$.

\section{Supplementary Files}

This is a list of supplementary files associated with this preprint. Click to download.

- suppTable1.docx 